ISSN 1997-5902

\title{
Épidémiologie de la consommation d'alcool par les femmes enceintes en Côte d'Ivoire : enquête sur 834 cas à Abidjan.
}

YAO Koffi Mathias, ASSI Bessekon Denis, BÂAbdoulaye, ADOU Kobenan Fiéni Jean-Baptiste, TAKO Nemé Antoine.

Laboratoire de Neurosciences, UFR Biosciences, Université Félix Houphouët Boigny de Cocody Abidjan. République de Côte d'Ivoire.

Correspondance email : yaomathias@gmail.com: Adresse : 22 BP 1676 Abidjan 22 / Téléphone : +225-08.71.58.80

Original submitted in on $3^{\text {rd }}$ June 2014. Published online at www.m.elewa.org on $31^{\text {st }}$ August 2014. http://dx.doi.org/10.4314/jab.v80i1.11

\section{RÉSUMÉ}

Objectif : Cette étude a eu pour but d'évaluer la proportion des femmes enceintes consommatrices d'alcool en Côte d'Ivoire.

Méthodologie et résultats : Une enquête sur les consommations d'alcool a recensé 834 femmes enceintes dans des centres de santé des dix communes de la ville d'Abidjan. Les résultats montrent que $37,8 \%$ des femmes ont consommé de l'alcool pendant la période de grossesse et, parmi elles, $11,4 \%$ en consomment dangereusement. Par ailleurs, $86,9 \%$ des femmes enquêtées sont mal informées sur les effets de l'alcool sur le fœtus. Aussi, les tranches d'âge les plus consommatrices sont celles allant de 26 ans à 35 ans (42,7\%) et celles de 36 ans à 44 ans $(43,9 \%)$ et les bières sont les boissons alcoolique les plus consommées.

Conclusion et application des résultats : Pour ces raisons, l'État devrait lancer une campagne pour sensibiliser les femmes ayant le potentiel d'avoir des enfants, sur les effets nocifs de l'alcool pendant la grossesse et pendant l'allaitement.

Mots-clés : femmes enceintes, alcool, consommation, Abidjan (Côte d'lvoire).

Epidemiology of alcohol consumption by pregnant women in Côte d'Ivoire: survey of 834 cases in Abidjan.

Objective: This study aimed to evaluate the proportion of pregnant women who consume alcohol in Côte d'Ivoire.

Methodology and results: A survey was conducted in health centers in the city of Abidjan. This survey recorded 834 pregnant women in the ten communes of Abidjan. This study shows that $37.8 \%$ of these women drank alcohol during pregnancy, and among the latter, $11.4 \%$ consumed it dangerously. In addition, $86.9 \%$ of the women who participated in the survey, were misinformed about the harmful effects of alcohol on the fetus. Furthermore, pregnant women who are age 26 to 35 and 36 to 44 were the biggest alcohol consumers and the beer was the most consumed.

Conclusion and application of results: On these grounds, the state should start a campaign to raise awareness among women of childbearing potential on the harmful effects of alcohol during pregnancy and during lactation. Keywords: pregnant women, alcohol , consumption, Abidjan (Côte d'Ivoire). 


\section{INTRODUCTION}

L'alcool semble reconnu comme la plus nocive des formes de pollution intra-utérine transmises par le comportement maternel (Abel et Sokol, 1987 ; Ebrahim et al., 1998). À l'occasion de toute consommation de boisson alcoolique pendant la grossesse, l'alcool traverse très facilement la barrière placentaire et sa concentration s'équilibre entre les compartiments maternel et fœtal, impliquant que la quantité d'alcool en contact avec les tissus du fœtus est rapidement comparable à celle acquise par la mère (Anonyme, 2001). La conséquence la plus grave de cette consommation est le syndrome d'alcoolisation fœtale (SAF), décrit pour la première fois par Lemoine en 1968 (Anonyme, 2001), puis par Jones et Smith en 1973 (Jacobson et Jacobson, 2002) comme étant une association de retard mental et de dysmorphie faciale. Le niveau de consommation sans risque n'est pas encore connu, de sorte que la recommandation la plus prudente reste l'abstinence pendant toute la grossesse et l'allaitement (Inserm, 2001). Si le minimum à consommer pour garder son fœtus en sécurité n'est pas connu, il est cependant

\section{SUJETS ET MÉTHODES}

Sujets : 834 femmes enceintes ont été sélectionnées dans les centres de santé des dix communes d'Abidjan, II s'agit de : 16 cadres, 82 employées subalternes et 736 femmes sans emploi fixe. Parmi elles, 465 vivent avec leur conjoint et les 369 autres vivent avec leur parent ou seul.

Méthodes: Pour réaliser ces enquêtes, un tirage aléatoire a été utilisé dans chacun des dix communes de la ville d'Abidjan. Dans chaque commune, un seul quartier est sélectionné et, dans chaque quartier, après avoir recensé les centres de santé fréquentés par les femmes enceintes, trois d'entre eux sont tirés de façon aléatoire. Tous les cas de grossesse des centres tirés sont concernés par l'enquête. Pour réaliser ce sondage, un questionnaire a été élaboré et traduit en langues vernaculaires (baoulé et dioula) pour les femmes qui ne comprenaient pas le français. Dix bénévoles ont été recrutés et formés pour la réalisation de ces enquêtes, dont le questionnaire est ainsi libellé :

1. Quel âge avez-vous?

2. Avez-vous une activité professionnelle ? Si oui, laquelle?

3. Consommiez-vous de l'alcool avant le début de prouver qu'au-delà de trois verres standards de consommation, la femme enceinte expose son futur enfant au SAF ou à d'autres anomalies liées à cette consommation abusive (Beaulac-Baillargeon, 2008). II faut souligner qu'un verre standard correspond à 13,6 grammes d'alcool pur, soit $341 \mathrm{ml}$ de bière à 5 degrés ou $142 \mathrm{ml}$ de vins à 12 degrés ou $43 \mathrm{ml}$ de spiritueux à 40 degrés (April, 2010). La prévalence des SAF et des autres anomalies liées à une consommation d'alcool par la mère n'est pas connue en Côte d'Ivoire, mais elle varierait entre 1,5 à 6 pour 1000 naissances en Amérique du nord (BeaulacBaillargeon, 2008). En Côte d'Ivoire, avec l'absence de sensibilisation, le faible niveau d'alphabétisation, la disponibilité des produits alcooliques, il existe un risque réel que les femmes enceintes ne s'abstiennent pas de consommer l'alcool. C'est pourquoi une étude épidémiologique est menée pour déterminer la proportion de femmes enceintes qui consomment de l'alcool. Par ailleurs, cette étude permettra d'évaluer le niveau d'informations de ces femmes et de comprendre leur comportement.

votre grossesse?

4. Consommez-vous de l'alcool pendant votre grossesse?

5. A quel rythme consommez-vous l'alcool ? Moins de 4 verres en moyenne dans le mois ?1 à 3 verres par occasion par semaine? Plus de 3 verres par occasion par semaine?

6. Saviez-vous que la consommation de l'alcool est déconseillée à la femme enceinte? Si oui, savez-vous pourquoi?

7. Quelles catégories de boissons alcooliques consommez-vous pendant votre grossesse? Les bières? Les vins? Les liqueurs?

Les données recueillies au cours de ces enquêtes sont traitées grâce au logiciel STATISTICA 10.0 qui a permis de faire des regroupements catégoriels. Ainsi, les différents groupes sont comparés au moyen du test de Khi2 (X2). La valeur limite inférieure du X2 acceptée est de 4 pour la significativité des différences (Schwart, 1978). Pour améliorer les approximations, le X2 avec la correction de Yates qui rend l'estimation plus prudente (HAYS, 1988) a été utilisée. En d'autres termes, si X2<4, la différence n'est pas significative; par contre, si $\chi 2 \geq 4$, 
la différence est significative et le degré de significativité $p$

\section{RÉSULTATS}

Niveau de consommation des femmes enceintes: Cette enquête montre que parmi les 834 femmes enceintes enquêtées, 315 d'entre elles (soit $37,8 \%$ ) ont consommé de l'alcool durant leur grossesse (Khi2 $=33$ et $p<0,0001$. La différence est très significative. Le degré de consommation est multiforme. En effet, certaines femmes consomment moins de l'équivalent 4 verres d'alcool dans un mois et en plusieurs occasions cumulées. Elles constituent un effectif de 84 (soit 26,7\% des consommatrices). Elles ont été classées dans le niveau 1 (risque mineur) de consommation. Les autres niveaux de consommation sont, d'une part, ceux du niveau 2 (risque moyen), au nombre de 195 (soit 61,9\%), qui correspond au groupe des femmes enceintes ayant consommé entre unà trois verres d'alcool par occasion et par semaine et d'autres part, celles du niveau 3 (risque accru), au nombre de $36(11,4 \%)$ qui consomment plus de trois verres par occasion et par semaine. En comparant les femmes ayant une consommation de niveau $1(26,7 \%)$ avec celles ayant une consommation de niveau $2(61,9 \%)$, on obtient un Khi2 $=30,27$ et $p<$ 0,0001 . Cette différence est très significative. En comparant le même groupe de niveau 1 au groupe des femmes du niveau 2 de consommation d'alcool $(61,9 \%)$, on obtient Khi2 $=15,4$ et $p<0,0001$. Cette différence est aussi très significative. Enfin, les consommatrices de niveau 2, avec une proportion de 61,9\%, comparée aux consommatrices de niveaux 3 montrent un Khi2 $=81,49$ et $p<0,0001$. La différence est donc très significative.

Niveau d'information, tranche d'âge des consommatrices : Les résultats montrent que sur les 834 femmes enquêtées, 109 d'entre elles (soit 13,1\%) était bien informées (figure1C) ; ce qui indique que les $86,9 \%$ étaient mal informées sur les risques de la consommation d'alcool pendant la période de grossesse $($ Khi2 $=321,32$ et $p<0,0001)$. Cette différence est très significative. La bonne information concerne surtout les risques encourus pour le fœtus durant toute la durée de la grossesse. II s'agit alors de souligner qu'aucune est inférieur ou égal à 0,05.

consommation n'est en principe admise puisque personne ne connaît la dose limite sans danger pour le futur enfant.

Tranche d'âges des consommatrices: Afin de déterminer les tranches d'âges les plus exposés à la prise d'alcool pendant la grossesse, un traitement statistique a été effectué en considérant quatre tranches d'âges. II s'agit des tranches d'âge de 14 à 18 ans, de 19 à 25 ans, de 26 à 35 ans et de 36 à 44 ans. Ainsi, 8,6\% des femmes de 14 à 18 ans consomment de l'alcool contre $29,5 \%$ chez les femmes de 19 à 25 ans ; le Khi2 = 4,32 et $p=0,04$. La différence est significative. En comparant la même tranche d'âges (14 à 18 ans avec $8,6 \%$ de consommation d'alcool) à la tranche d'âges de 26 à 35 ans avec une proportion de $42,5 \%$ de consommatrices d'alcool, on obtient un Khi2 $=8,13$ et $p=0,004$; la différence est aussi significative. De même, en comparant cette même tranche d'âges (14 à 18 ans) à celle des femmes de 36 à 44 ans $(19,4 \%$ de consommation), on observe que Khi2 $=6,98$ et $p=0,008$. Cette différence est aussi significative. Par contre, 29,5\% des femmes enceintes de la tranche d'âge de 19 à 25 ans consomment de l'alcool. En les comparant à celles de la tranche d'âge de 26 à 35 ans, avec une proportion de consommation d'alcool de $42,5 \%$, on trouve Khi $=0,83$ et $p=0,36$. Cette différence n'est pas significative. De même, la comparaison du niveau de consommation d'alcool des femmes enceintes de la tranche d'âge de 19 à 25 ans, avec une proportion de $29,5 \%$, et celles de la tranche de 36 à 44 ans, avec une proportion de 19,4\%, donne un Khi2 $=0,72$ et $p=0,40$. Cette différence n'est donc pas significative. Enfin, en comparant la tranche d'âge de 26 à 35 ans, avec un niveau de consommation d'alcool à $42,5 \%$ à celle de 36 à 44 ans dont le niveau de consommation d'alcool est à $19,4 \%$, on obtient Khi $2 \approx 0$ et $p=0,95$. Cette différence n'est également pas significative. 

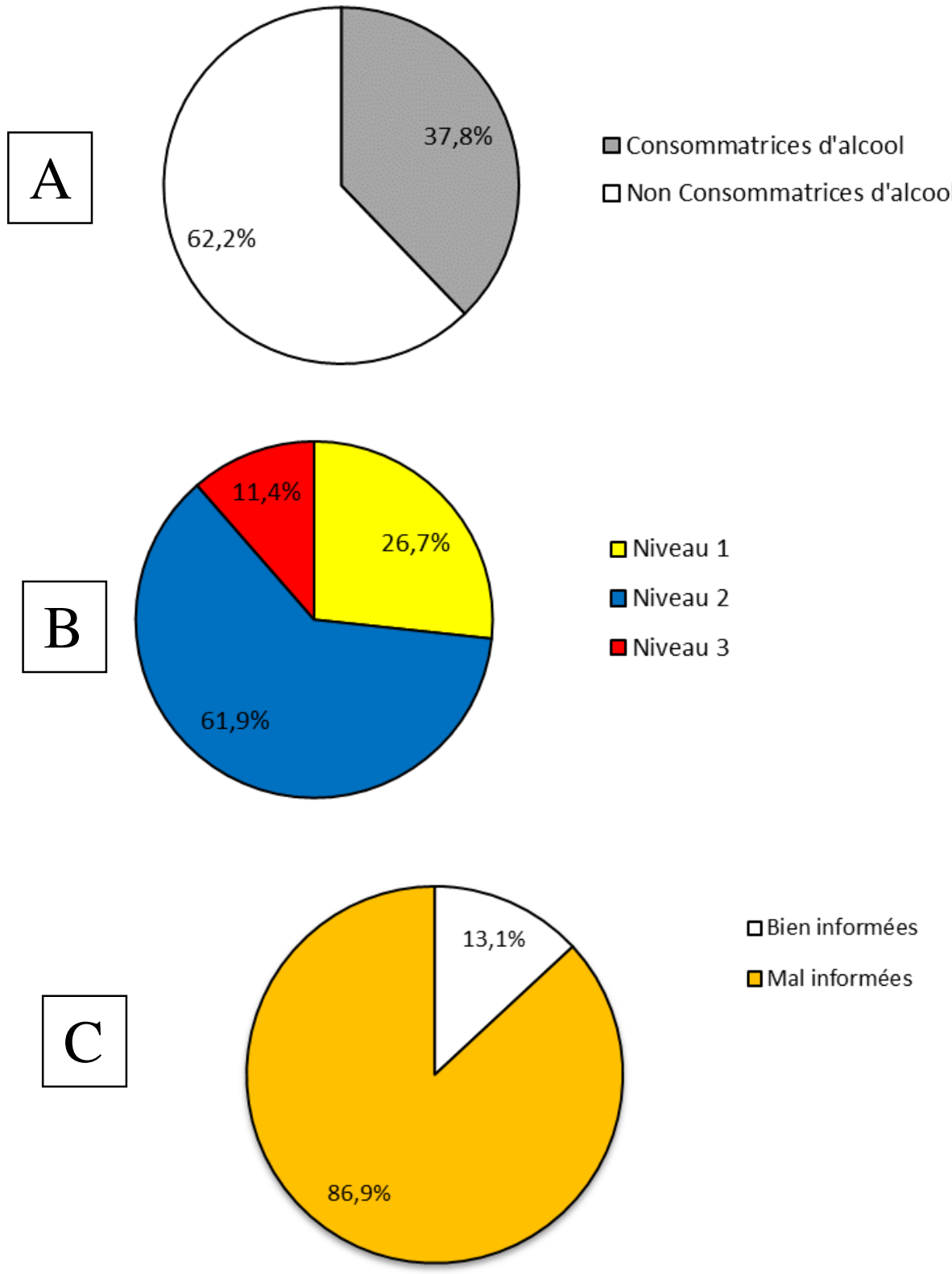

Niveau 1 : Moins de 4 verres par mois en plusieurs occasions.

Niveau $2: 1$ à 3 verres par occasion et par semaine.

Niveau 3 : plus de 3 verres par occasion et semaine.

Figure 1 : Vue d'ensemble de la consommation d'alcool par les femmes enceintes :

en $\mathrm{A}$, la proportion des consommatrices par rapport aux non consommatrices ;

en $B$, la répartition des niveaux de risque encouru ;

et en $\mathrm{C}$, la proportion des femmes ayant reçu de bonnes informations ou pas, concernant l'alcool et la grossesse. 


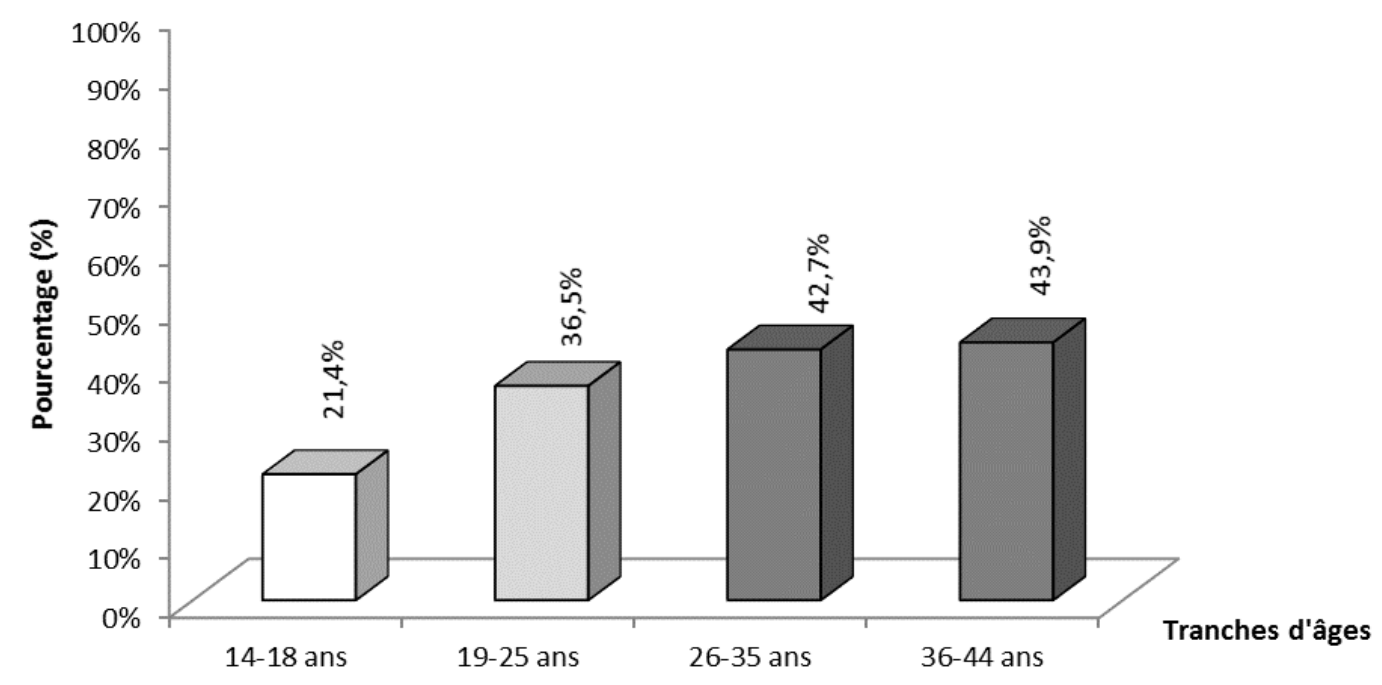

Figure 2 : Répartition des tranches d'âge des femmes enceintes consommatrices d'alcool. Les plus grandes consommations concernent les tranches d'âge de 26 à 35 ans et de 36 à 44 ans.

Types de boissons alcooliques consommées : Les différents types d'alcool sont regroupés dans la catégorie des bières, la catégorie des vins et la catégorie des liqueurs (figure 3). La comparaison des consommations des femmes donnent les résultats suivants : $81 \%$ des femmes consommatrices ingèrent les bières et $23,8 \%$ consomment les vins (Khi2 $=65,65$ et $p<0,0001$ ) cette différence est très significative. Aussi, en comparant la consommation des bières à celle des liqueurs $(11,7 \%)$, on obtient Khi2 $=116,22$ et $p<0,0001$. Cette différence aussi très significative. Par ailleurs, entre la consommation des vins $(23,8 \%)$ et celle des liqueurs $(11,7 \%)$, on obtient Khi2 $=10,31$ et $p=0,0013$. Cette différence est également significative.

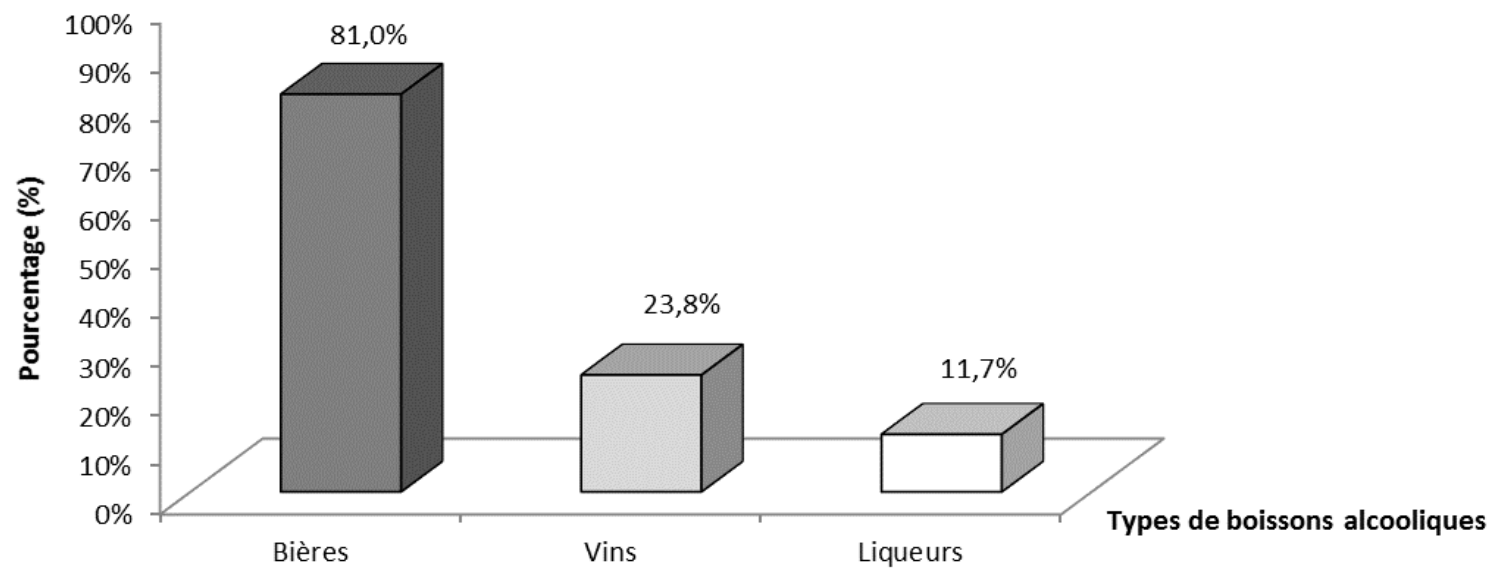

Figure 3 : répartition de la consommation des boissons alcooliques par les femmes enceintes. Les bières sont les plus consommées par les femmes enceintes. 


\section{DISCUSSION}

Les résultats de cette enquête estiment à $37,8 \%$, la proportion de femmes enceintes qui s'adonnent à la consommation d'alcool. Cette proportion, bien que significativement faible par rapport aux femmes enceintes non consommatrices $(62,2 \%)$, constitue un motif d'alerte. En effet, ces valeurs reflètent le niveau de consommation habituellement rencontré chez les femmes dans les enquêtes générales déjà effectuées en Côte d'Ivoire (Yao, 2009; Yao et al, 2012). Cela montre que les femmes enceintes ne prennent aucune disposition particulière par rapport à leur consommation d'alcool en relation avec leur état de grossesse. Ce résultat montre que contrairement à certains pays comme la France qui connait une baisse importante de la proportion de consommatrices en état de grossesse (11\%), les femmes vivant en Côte d'lvoire ne sont pas suffisamment informées. En effet, une étude française indique que la consommation globale des femmes en France serait de 38\% (Saurel-Cubizolles et al., 2010). De même, en Côte d'Ivoire, les études récentes indiquent que la consommation féminine serait de 41\% (Yao et al., 2012). La grande faiblesse de la proportion de consommatrices enceintes en France par rapport à la Côte d'Ivoire serait due, certainement, à un ensemble de mesures qui permettent à ces femmes en France d'être mieux informées. Cette déduction se trouve renforcée par une étude Canadienne sur les proportions de femmes enceintes consommatrices d'alcool. Cette étude révèle que $10,5 \%$ des femmes enceintes au Canada consomment de l'alcool pendant leur grossesse alors que la proportion de femmes non enceintes consommatrices serait de $62,4 \%$ (Chudley et al., 2005). Les résultats de la présente enquête indiquent que $86,9 \%$ des femmes enceintes enquêtées sont mal informées ou n'ont aucune information relative à leur consommation d'alcool pendant la grossesse. Ce constat montre, par ailleurs, qu'à Abidjan, les services de santé ne sensibilisent pas suffisamment (ou pas du tout) les femmes enceintes sur les consommations d'alcool. Par ailleurs, si les recherches n'ont pas encore pu indiquer quel serait le niveau de consommation d'alcool sans risque pour le fœetus, il est montré qu'au-delà de trois verres standards par occasion, le fœtus serait en danger, surtout si cette

\section{CONCLUSION}

Cette étude montre que le niveau de consommation des femmes enceintes, en Côte d'Ivoire, est élevé. Cette consommation dangereuse serait en rapport avec le manque d'informations et concernerait beaucoup plus la catégorie des bières. C'est pourquoi, l'État doit engager consommation est répétée plusieurs fois durant la grossesse (Anonyme, 2001). Dans cette recherche, celles qui consommaient avec un risque très accru (niveau 3) constituent $11,4 \%$ des consommatrices. Ce taux semble assez élevé s'il devrait correspondre au nombre d'enfants ayant des séquelles due à la consommation d'alcool de la mère. Par ailleurs, la proportion de consommatrices du niveau 2 (risque moyen) est aussi alarmante avec $61,9 \%$. En effet, la fragilité du fœtus, avec le développement continuel des organes, la multiplication rapide des cellules, impliquent qu'aucune consommation n'est bénéfique pour le fœtus (Randall, 2001; Kartik et Harihara, 2005), même si certains auteurs comme O'Brein (2007) soutiennent la possibilité de petites consommations sans risque. Cependant les recherches de ces auteurs ne précisent pas la dose minimale acceptable par le fœtus sans dommage. Aussi, il a été démontré que plusieurs autres facteurs influenceraient le risque réel de développer, chez l'enfant, un trouble causé par l'alcoolisation fœtale. Parmi ces facteurs, il y a les quantités et les doses d'alcool ingérées, la fréquence d'ingestion, des facteurs génétiques, nutritionnels et environnementaux, l'âge de la mère (Lockhart 2001; Jacobson et Jacobson, 2002 ; Warren et Li, 2005). Cette étude a voulu identifier la tranche d'âge la plus concernée par cette consommation risquée d'alcool. Les résultats montrent que la tranche d'âge de 14 à 18 ans était significativement la moins consommatrice et que les femmes enceintes les plus consommatrices sont celles de 26 à 44 ans. La faible proportion des consommatrices de 14 à 18 ans serait due aux faibles moyens financiers dont disposeraient ces jeunes filles enceintes. Inversement, les autres tranches d'âge constituent l'ensemble des femmes qui ont un soutien financier dans la société, soit par leur conjoint ou par leur propre travail (Bondel et al., 2012). Par ailleurs, les types de boissons alcooliques concernées par la consommation à risque des femmes enceintes restent dominés par la catégorie des bières. Ce résultat est en accord avec les données obtenues par May et Gossage (2001) dans une enquête sur le même sujet au ÉtatsUnis.

une politique de prévention par la sensibilisation des femmes en âge de procréer et, particulièrement des femmes enceintes. Pour être efficaces, les messages de prévention doivent être clairs, simples, non anxiogènes, permettre de comprendre quel comportement il est 
souhaitable d'adopter et quels sont les comportements de consommation d'alcool qui sont plus à risque durant la grossesse (Eriksson, 2007). Pour le moment, au vue des avancées scientifiques, les mesures adoptées par plusieurs pays en la matière consistent à déconseiller la consommation d'alcool pendant toute la période de la grossesse « Zéro alcool pendant la grossesse ». Par

\section{RÉFÉRENCES BIBLIOGRAPHIQUES}

Anonyme, 2001. Alcool : effets sur la santé. Expertise collective INSERM (Institut National de la Santé et de la Recherche Médicale); France, Septembre 2001. 327pp.

April N, 2010. Alcool et grossesse : épidémiologie, risque et recommandations aux femmes enceintes. Journées annuelles de santé publique, Québec 24 novembre 2010 ; 28p.

Beaulac-Baillargeon L, 2008. De la mère au nourrisson. La prise d'alcool pendant la grossesse et l'allaitement. Québec Pharmacie 55(7) : 13-18.

Blondel B, Lelong N, Kermarrec M, Goffinet F, 2012. Coordination nationale des enquêtes nationales périnatales. La santé périnatale en France métropolitaine de 1995 à 2010. Résultats des enquêtes nationales périnatales. J Gynecol Obstet Biol Reprod 41: 151-66.

Carson G, Cox LV, Crane J, Croteau P, Graves L, Kluka S, Koren G, Martel MJ, Midmer D, Nulman I, Poole N, Senikas V, Wood R, 2010. Directive clinique de consensus sur la consommation d'alcool et la grossesse. Journal d'obstétrique et gynécologie du Canada 32(8) (suppl 3) : S5-S9.

Chudley AE, Conry J, Cook JL, Loock C, Rosales T, LeBlanc N, 2005. Ensemble des troubles causés par l'alcoolisation fœtale: lignes directrices canadiennes concernant le diagnostic. CMAJ $172, n^{\circ} 5$ (suppl.), S2-S21pp.

Eriksson UJ, 2007. Fetal ethanol exposure during pregnancy - how big is the problem and how do we fix it? Acta Paediatr 96(11): 1557-1559.

Jacobson JL and Jacobson SW, 2002. Effects of prenatal alcohol exposure on child development. Alcohol Research \& Health 26(4): 282-6.

Lockhart PJ, 2001. Fetal alcohol spectrum disorders for mental health professionals: A brief review. Current Opinion in Psychiatry 14: 463-469.

May PA and Gossage JP, 2001. Maternal risk factors for fetal alcohol spectrum disorders. Not as simple as it might seem. Alcohol Research \& Health 34(1): 15-26. exemple, en France, en 2006, un arrêté préconisait la présence sur toutes les bouteilles d'alcool d'un pictogramme représentant une femme enceinte, un verre à la main, barrée d'un trait rouge, pour informer les femmes des dangers liés aux boissons alcoolisées et pour illustrer la campagne « Zéro alcool pendant la grossesse » (Saurel-Cubizolles et al., 2010).

O'Brien P, 2007ls it all right for women to drink small amounts of alcohol in pregnancy? Yes. BMJ 335: 856.

Randall CL, 2001. Alcohol and pregnancy: Highlights from three decades of research. Journal of Studies on Alcohol 62: 554-561.

Saurel-Cubizolles MJ, Prunet C, Blondel B, 2013. Consommation d'alcool pendant la grossesse et santé périnatale en France en 2010. Bulletin Epidémiologique Hebdomadaire, $\mathrm{n}^{\circ} 16,17,18$ : 180-197.

Shankar K and Mehendale HM, 2005. Fetal Alcohol Syndrome in BAnderson, A de Peyster, S Gad, P.J. Hakkinen, M Kamrin, B Locey, H Mehendale, C Pope, L Shugart. Encyclopedia of toxicology, vol2. Academic press, 2nd eds., pp 330-331.

Warren KR and Li TK, 2005. Genetic polymorphisms: Impact on the risk of fetal alcohol spectrum disorders. Birth Defects Research. Part A. Clinical and Molecular Teratology 73(4): 195203.

YAO KM, 2009. Approche épidémiologique de la consommation d'alcool en Côte d'lvoire et évaluation des effets de l'alcoolisation (aigue et chronique) au koutoukou (eau-de-vie de vin de palme) sur le fonctionnement cérébral des consommateurs. Thèse de doctorat en Physiologie Animale, Université de CocodyAbidjan ; N593, 151pp.

Yao KM, Camara PA, Adou KFJB, 2012. Types de boissons alcooliques consommées en Côte d'lvoire. Préférence et consommation effective. Alcoologie et Addictologie ; 34(3) : 185-193. 